\title{
Development board of the autonomous semi-passive RFID transponder
}

\author{
P. JANKOWSKI-MIHUŁOWICZ*, M. WĘGLARSKI, G. PITERA, D. KAWALEC and W. LICHOŃ \\ Department of Electronic and Communications Systems, Faculty of Electrical and Computer Engineering, \\ Rzeszów University of Technology, W. Pola 2, 35-959 Rzeszów
}

\begin{abstract}
The idea of multiband development board designed to support and advance the technique of battery-less autonomous semi-passive RFID transponders is presented in the paper. The extra function of harvesting energy from the electromagnetic field of different radio communications systems, the ultra efficient power conditioner and the energy storage block are applied in the proposed construction. The facility of utilizing gathered energy is provided to support designers in developing applications of automatic object identification. Since the evaluation board is to work without a galvanic cell, the particular emphasis is put on the mechanisms that improve energy efficient operation of the whole electronic circuit. The construction principles and operation modes (charging, cold start, sleep with charging, sleep, measurement and cut-off) of the storage unit are described in depth in the paper. The preproduction batch of the demonstrators has been manufactured on automated assembly line at ELMAK Ltd. Company, according to the elaborated model. It confirms that the author's demonstrator of the battery-less autonomous semi-passive transponder can be reproduced on an industrial scale. On the basis of the preproduction series, the performance parameters of the final product have been estimated. The presented study should significantly contribute to the RFID technique therefore the authors set up the evaluation process of marketing research and product commercialization.
\end{abstract}

Key words: RFID, semi-passive transponder, energy harvesting, sensors, development board.

\section{Introduction}

An increasing number of implemented radio frequency object identification systems confirms the usefulness of radio frequency identification (RFID) technique [1]. With regard to economic aspects, the growth in this electronics sector is due to the rising availability of RFID devices in the market and also long-term forecasts of automatic identification more common use in the coming years [2]. When the technical capabilities are taken into consideration, the RFID development is caused by research activity in this scope - progress in understanding: principles of the system operation [3], problems of estimating RFID device parameters (some of problems in RFID technique cannot be solved by using laws known from the classical theory of antennas) $[4,5]$, restrictions in users' applications, etc.

The widely understood processes of the electronic identification in numerous areas affecting the daily lives and economy [1-3, 6, 7] are a matter of intensive commercialisations. Significant number of the implementations is carried out in the range of the Internet of things. The RFID system complied with electronic product code (EPC) recommendations is thought to replace barcodes being currently in common use. The development works are conducted to ensure that automatic identification will be effectively and smoothly applied to fast moving consumer goods (FMGC) in the world supply chain [6]. Similar activities are pursued in the areas of reliable and safe identi-

*e-mail: pjanko@prz.edu.pl fication of moving objects, e.g. in the public transport (AVI - automatic vehicle identification) [7].

So it can be concluded that the observed applicable potential of the RFID technology justifies the need to develop new designs of electronic transponders. New more sophisticated solutions of electronic tags will be a factor of innovative progress in the discussed scope of use.

\section{Transponder in RFID system}

In terms of hardware (Fig. 1), an RFID system consists of a read/write device (RWD), which includes a transmitter (TX) and a receiver $(\mathrm{RX})$, its antenna and at least one electronic transponder that is intended for marking an object. Communication in this system (read/write data) is provided with one (single system) or with multiple transponders simultaneously (anti-collision system). The marked objects during the identification process may be placed in fixed points (static system) or may dynamically change their location (dynamic system). The interrogation zone (IZ) is the main parameter of the RFID system, because it determines the efficiency of a given automated process [5]. The size of the IZ strongly depends on the above mentioned arrangements of RWD and transponders.

According to the main classification of the RFID systems it is possible to distinguish applications operating in the inductive or radiative coupling regime. In the first group, the carrier frequency $f_{0}$ is between $100 \mathrm{kHz}$ and $135 \mathrm{kHz}$ (typically $125 \mathrm{kHz}$ ) for the LF band or $13.56 \mathrm{MHz}$ of the HF band whereas in the 


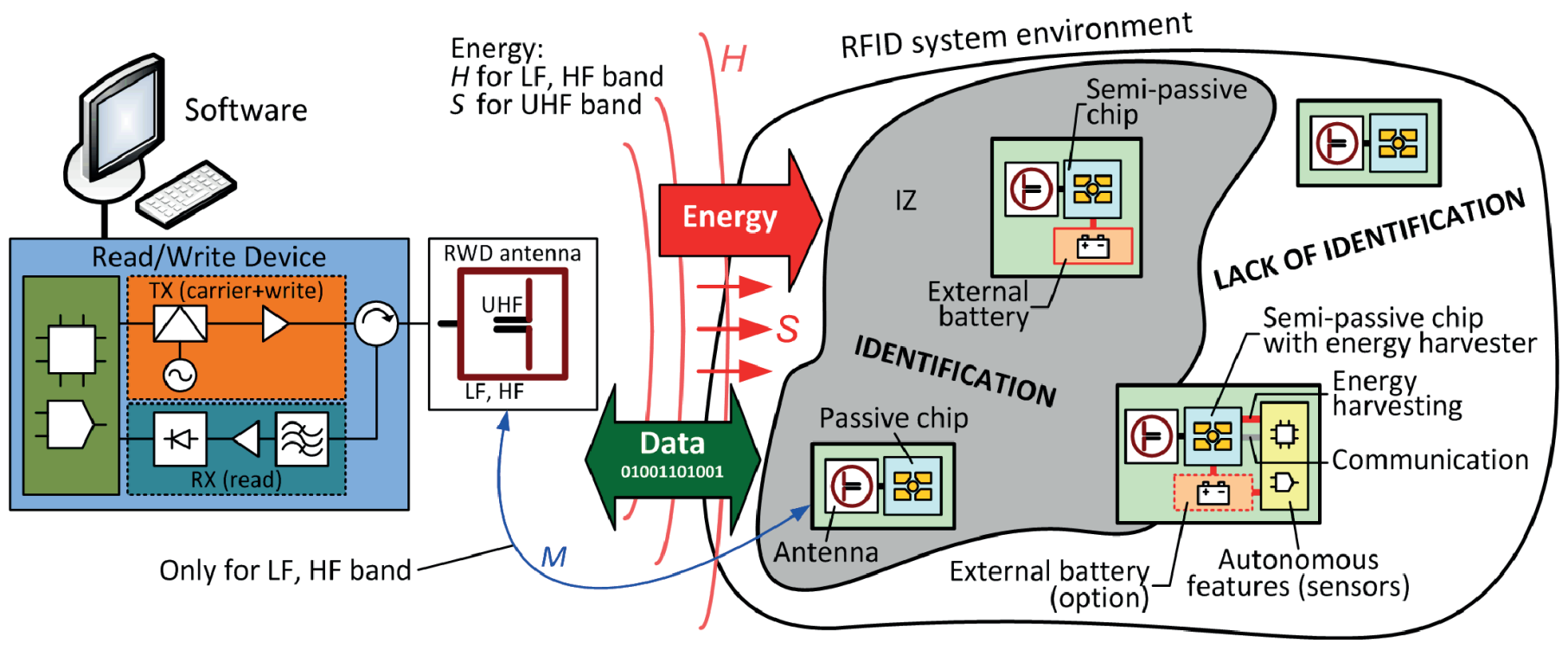

Fig. 1. Block diagram of an RFID system

second group - from $860 \mathrm{MHz}$ to $960 \mathrm{MHz}$ (the UHF band) depending on the region of the world [3]. The inductive coupled systems operate by utilizing zone that is characterized by an inhomogeneous magnetic field (described by the magnetic field strength $H$ ) and strong coupling (described by the mutual inductance $M$ ) between antennas of the arrangement components [8]. On the other hand, the UHF RFID systems work in the range of far-field where emitted wave is not only a data carrier but first of all an energy source (of power density $S$ ) [5]. The RWD antenna together with transponder antennas comprise a radio communication arrangement that has to be wave and impedance matched [4]. In the all frequency bands, the communication mechanisms are implemented in adequate protocols that are typically defined by the ISO/IEC standards (e.g. for the HF band: ISO/IEC 15693, 14443 or 18000-3, for the UHF band: compatible with EPC Class 1 Gen 2 - ISO/IEC 18000-6).

The most popular passive RFID transponder contains only the chip with the connected antenna (Fig. 1). Access to the internal memory of the chip can be done only by using the radio communication interface. Semi-passive (sometimes called active) RFID transponders have built-in an extra supply source (e.g. lithium disposable battery) which can be exchangeable or not. Generally it is used for enlarging the IZ, what is a very desirable feature for most implementations of RFID systems. Moreover, in some new chips integrated with both wired and wireless access ports [5, 8], the additional energy is used for powering blocks of supplementary autonomous functions, such as measuring physical quantities (humidity [9-10], temperature [10-12], light intensity [10,11], pressure [13], acceleration [14], gas [12], etc.), writing gathered data into a built-in memory, managing activity cycles and power distribution in a data acquisition system. These functions are realised without the participation of RWD devices and give transponders the autonomy.

The first new untypical RFID chips with the possibility of gathering extra energy from the electromagnetic (EM) field emitted by RWD antennas have been just announced in the contemporary electronic market. Usually, these very rare solutions can operate in a semi-passive mode (with battery) or in a passive mode (without battery). However, it should be kept in mind that the chips with the ability of recovering energy require a different antenna pattern than it is in the conventional passive transponders [15]. It should be also noted that the RWD has to be still active to conduct the radio communication process (transmission data in semi-passive RFID systems), because the extra battery system can never be used for activating the transmission circuit. It means that the transponder antenna does not emit the EM field as it is in the case of conventional short range devices (SRD) [16]. These characteristics help distinguish the semi-passive RFID transponders from the classical active SRDs.

\section{Idea of battery-less autonomous semi-passive RFID transponders}

A small application area of commercial semi-passive transponders compared to the passive constructions is due to disadvantages of the electrochemical cells. The commonly used batteries have a limited lifetime decreasing in harsh environmental conditions (e.g. in low temperature ambient), restricted ability to supply a load with short high-power pulses or they have to be replaced after discharge, protected against theft, etc. Because of these drawbacks, the rare semi-passive RFID systems are expensive in production and maintenance, not stable and do not provide a high level of identification efficiency. To cope with these inconveniences, the authors work out the multiband development board dedicated to battery-less autonomous semi-passive RFID transponders in which the extra functions of energy harvesting from the electromagnetic field of different radio communications systems as well as 
the dedicated power conditioner and the energy storage block are implemented.

The proposed idea contributes significantly to the automatic identification development (Fig. 2). The beginning stage of the progress in the identification system - optical machine-readable barcodes - provides only basic and unchangeable information about marked objects. The recognition process can be realised only with a single object simultaneously and the barcode has to be visible to a reader. Currently, passive and semi-passive (but not autonomous) RFID transponders are beginning more and more popular and they mark a new stage in the advancement of object identification. They can be not only read but also it is possible to change the stored data during operational use of the systems, configuration, maintenance, etc. Moreover, some user's extension information is written into the tag memory and the read/write process can be realised simultaneously with several transponders (anti-collision identification) without optical visibility.

The author's developed idea helps to open a new chapter in the automatic object identification. In the third stage, the RFID transponders provide not only details about marked objects but also extended variable data gathered from surroundings by built-in sensors of different physical quantities. Moreover, the transponders are developed towards the capability of being powered from the electromagnetic field of common radio communications systems. In this case, the internal supply consists of an energy harvester integrated with an RF front-end and an advanced low voltage regulator supported by a super-capacitor. The storage block of environmental energy is free of drawbacks and limitations that are inherent for the traditional galvanic cells $[17,18]$. The super-capacitors are maintenance-free, with low degree of wear. Their high capacity at their small dimensions bridge the gap between possibilities of electrolytic capacitors and rechargeable batteries - significant amount of energy can be released with high volume power [19].

Another important problem is to provide suitable conditions for charging the untypical power source of the autonomous semi-passive transponders. There are examples of effective using electromechanical, thermal or photovoltaic transducers $[20,21]$ for obtaining energy from the environment. But in the view of RFID system operation principles, the electromagnet-

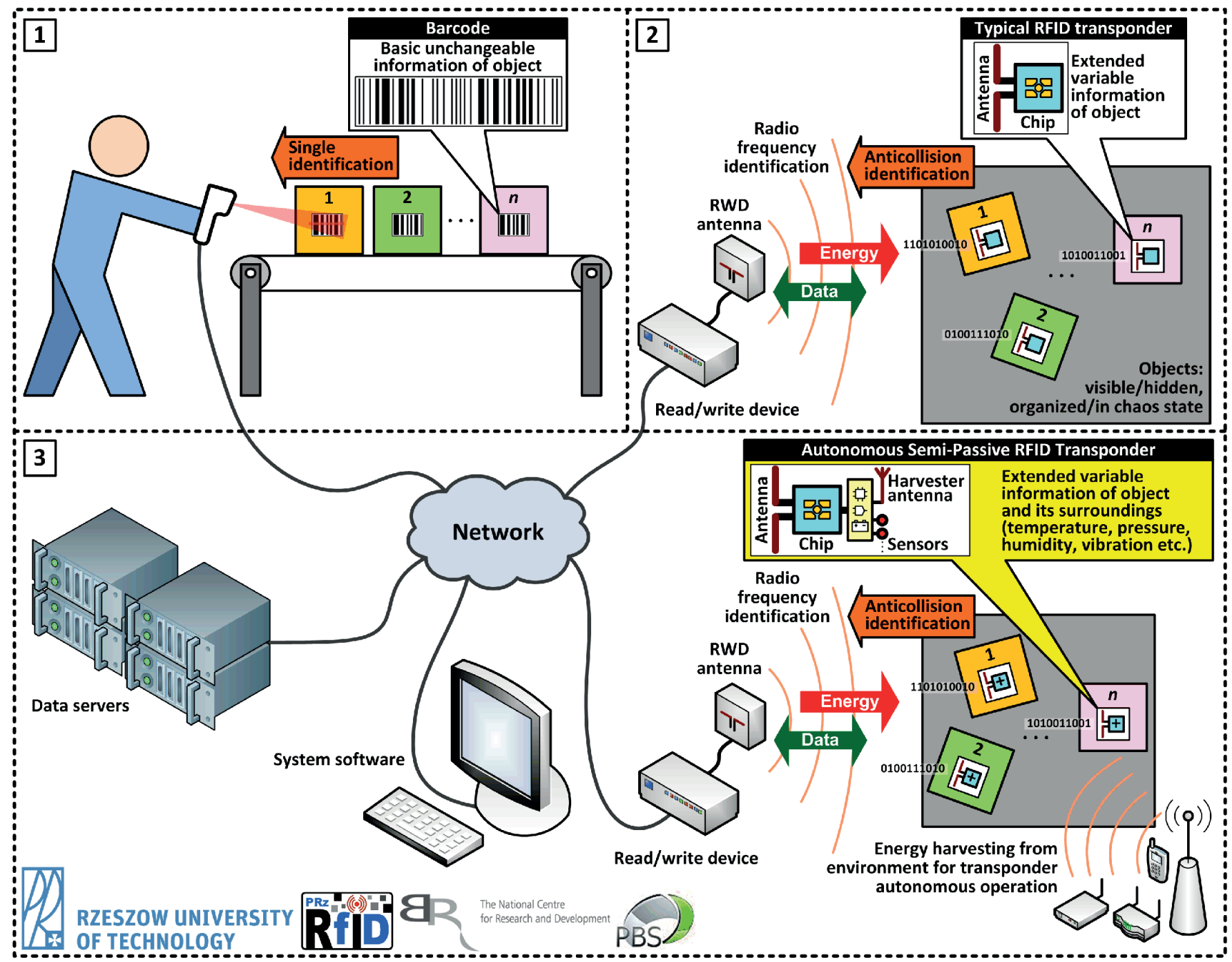

Fig. 2. Development of automatic object identification systems 
ic field generated by radio communications systems (e.g. base transceiver stations of the wireless communication technologies like GSM, wireless local loop, Wi-Fi, WiMAX or other wide area networks, etc.) that are present almost in every place on the Earth is a more natural source. The choice of electromagnetic waves as the medium of conveying power in the proposed autonomous development board follows the new solutions that appear in RFID chips of semi-passive transponders - a few producers offer the possibility to split energy supplied by the RWD to the chip between the internal communication transceiver and additional blocks e.g. sensors, analog-to-digital converters, external outputs, etc $[5,8]$. Although the foreign electromagnetic field presents in the surroundings supports new transponder functionality of operating autonomously outside the IZ, it is very weak and problems of capturing, storage and converting very low energy to the usable level has to be solved.

The proposed research conception will hopefully make a real contribution towards easing integration of low power devices in the transponder structure and it allows the semi-passive transponders to be fully autonomous. In fact, it should be stated that commercial potential of such possibilities has been described in the literature but it practical usefulness in RFID system applications is significantly restricted by the described drawbacks of galvanic cells that are presently in use.

\section{Multiband development board of battery-less autonomous semi-passive RFID transponder}

The proposed conception has been realised under the Polish government project of the PBS1/A3/3/2012 number. The multiband development board of battery-less autonomous semi-passive RFID transponder has been elaborated on the basis of worked out assumptions. The electrical harvester from the electromagnetic field of different radio communications systems, sophisticated low voltage converter and low leakage energy storage have been designed especially for the demonstrator (Fig. 3).

All electronic circuits are built on the two-sided copper-clad PCB laminate ISOLA IS680-300 (dielectric thickness: $1.547 \mathrm{~mm}$, copper layer thickness: $18 \mu \mathrm{m}$, relative dielectric permittivity: $\varepsilon_{r}=3.08$ at $1 \mathrm{GHz}$, dielectric $\operatorname{loss} \operatorname{tg} \delta=0,003$ [22], PCB board dimensions: $115.94 \times 89.44 \times 1.547 \mathrm{~mm}$ ) (Fig. 4$)$.

The demonstrator is equipped with two independent interfaces of object radio identification - one of them operates in the HF band (ISO IEC 15693 protocol) and the second in the UHF band (EPC Class 1 Gen 2 protocol consistent with ISO IEC 18000-6c). These blocks are designed on the basis of semi-passive chips that have additional wire serial peripheral bus for communication with a supervising controller (HF band: ST M24LR64E chip with I2C bus; UHF band: chip AMS SL900A, SPI bus) $[5,8,15]$. Moreover, the both ICs can gather energy from the electromagnetic field generated by antennas of RWD, in their operating frequency bands. It means that the super-capacitor of the supply unit is charged whenever the demonstrator appears in the IZ. Of course, the communication in RFID system can be realised without regard to activities of the extra blocks.

The autonomy of the semi-passive transponder is achieved by applying a harvester that gathers environmental energy derived from radio communications systems present in the research laboratory location. The antenna of special construction is designed for the presented demonstration board. It operates in the frequency band of 930-975 MHz (VSWR $<2$ ) and is matched to the input circuit of the Powercast P2110B harvester. The block provides the RF harvesting from GSM900, converts RF energy to DC and stores it in a capacitor. Also the power management for battery-free micro-power devices is implemented. It is possible to measure the value of power at the input and to adjust how frequently the module is woken up depending on the existing energy conditions. The research and development cooperation with Powercast Company allows the authors to redesign the development board to another radio communications system (e.g. UMTS, LTE, WiFi).

The typical environmental RF energy source is characterized by low capacity [23] and it causes the necessity to design an ultra efficient energy storage unit (ESU). The built-up module is consisted of two ultra efficient boost (step up) switching regulators (Silicon Labs TS3310), super-capacitor (Cellergy CLC03P025F12), ultra low ON-resistance load switch with controlled turn on with hysteresis (Texas Instruments TPS22934)

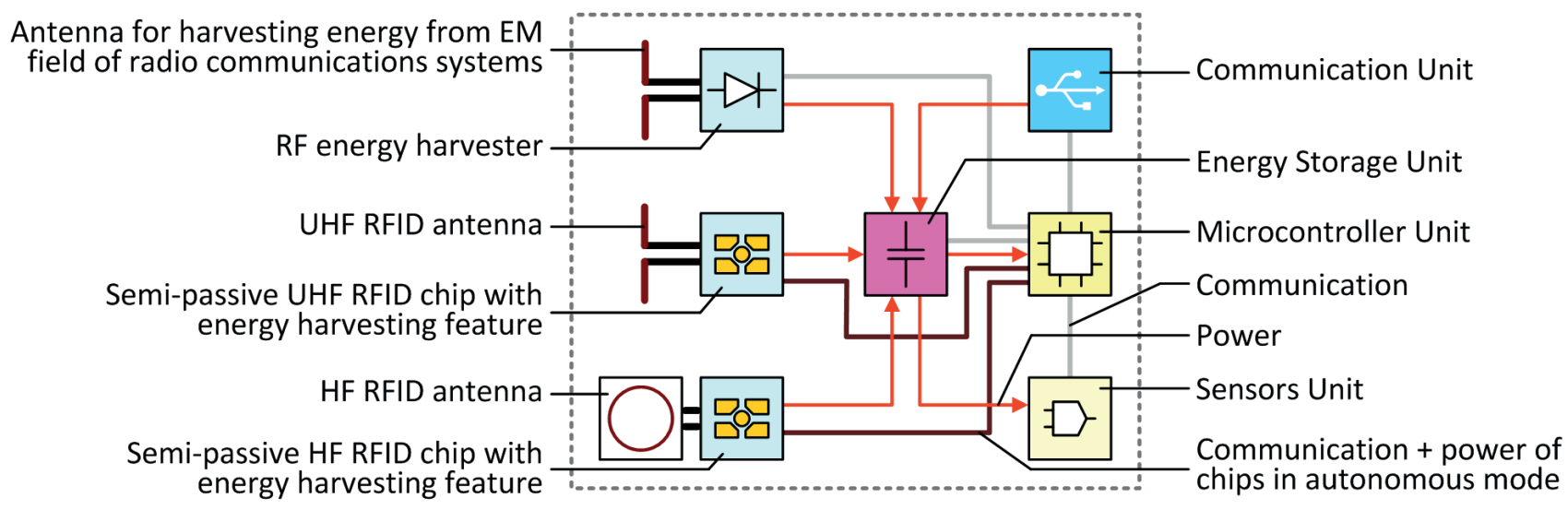

Fig. 3. Block diagram of development board 


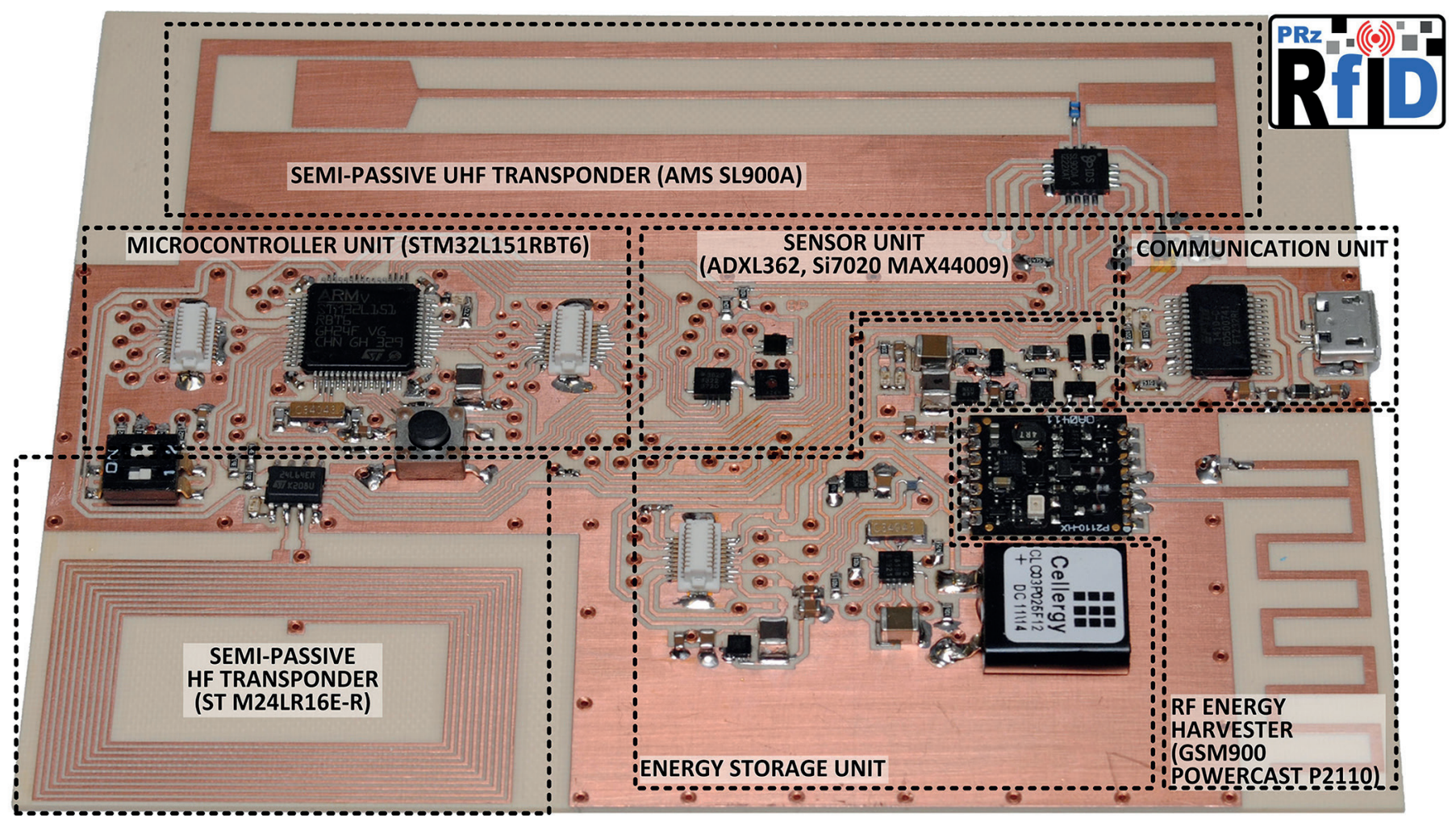

Fig. 4. Elaborated development board

and low power real time clock (RTC Abracon AB1815). Its block diagram and steps of operation are presented in Fig. 5. The DC-DC boost converters provide conditioned voltage at switched output that completely isolates loads from the storage capacitor. They improve the efficiency of energy harvesting since the super-capacitor can be fully charged even in the presence of weak electromagnetic field. It helps also to prevent the microprocessor control system ( $\mu \mathrm{C}$ unit) from turning on till the time when the reservoir of energy reaches the sufficient level - the activity management is supported by the key with hysteresis. An additional key controlled by the real time clock is used to execute operation in short-time duty mode - it cuts off the supply of microcontroller and sensors and as a result saves energy and expands the operation time.

The applied Silicon Labs TS3310 is an ultra efficient boost converter dedicated to work in battery powered applications, with under voltage lockout (UVLO) feature at $0.85 \mathrm{~V}$ with a $0.02 \mathrm{~V}$ hysteresis. Its causes that the input of the converter can be automatically impedance-matched to the substitute circuit of the harvester blocks. The whole process of the energy conditioning, conversion and accumulation can be divided into a few main stages. At the beginning, the internal capacitors are assumed to be discharge, thus the first step (Fig. 5a) consists in charging the storage super-capacitor (its capacitance is equal $C_{S}=25 \mathrm{mF}$ ). The maximal amount of energy that is stored in this element is described by the formula:

$$
E_{\text {full }}=\frac{C_{S} \cdot U_{\text {Cmax }}{ }^{2}}{2}=\frac{25 \mathrm{mF} \cdot(3 \mathrm{~V})^{2}}{2}=112.5 \mathrm{~mJ}
$$

where: $U_{C \max }$ means the maximal voltage that is set up at the output of the DC-DC boost converter.

It is important that all functional blocks of the demonstrator are not active when the super-capacitor is charged. The microcontroller is activated by the $V_{D D}$ power line when the voltage reaches the value of turning on the switch (with the hysteresis: $U_{\text {Con }}=2.35 \mathrm{~V}$ ). It means that the level of gathered energy is enough for proper operation of the subsequent parts in the development board. In the presented solution this value equals:

$$
E_{\text {on }}=\frac{C_{S} \cdot U_{C o n}{ }^{2}}{2}=\frac{25 \mathrm{mF} \cdot(2.35 \mathrm{~V})^{2}}{2}=69.03 \mathrm{~mJ}
$$

The value of turn-on voltage depends on the parameters of the used switch (Texas Instruments TPS22934 in the presented demo board). The voltage hysteresis on the control input prevents microcontroller and sensor blocks against starting up too quickly, without sufficient power to finish tasks.

When the switch is active then the next converter is turn on. This block provides stable conditions of powering microcontroller and sensors. It also initiates the first measurement cycle (Fig. 5b). When the measurement data are acquired, the microcontroller is cut off by the switch controlled by the RTC circuit. If the field strength is still enough, the process of harvesting energy is pursued till the time when the super-capacitor will be fully charged (Fig. 5c). When the storing process is complete, the harvesting front-end is unloaded and the excess energy is used only to compensate losses in the circuitry. If the demonstrator is moved to the weak electromagnetic field, then 
it is powered from the super-capacitor and the process of its discharging starts (Fig. 5d).

The demonstrator works periodically. After the assumed period of time delay, it is woken up by the switch controlled by the RTC (Fig. 5e). Then the measurement cycle is performed and the memories of the microcontroller and RFID chips are synchronised. When the all tasks are finished, the demonstrator goes to sleep mode (Fig. 5d).

When the electromagnetic field is weak, the super-capacitor is discharged by the subsequent activity cycles of autonomous functions and the switch with hysteresis is turned off as a consequence (Fig. 5f). Nevertheless, there is certain amount

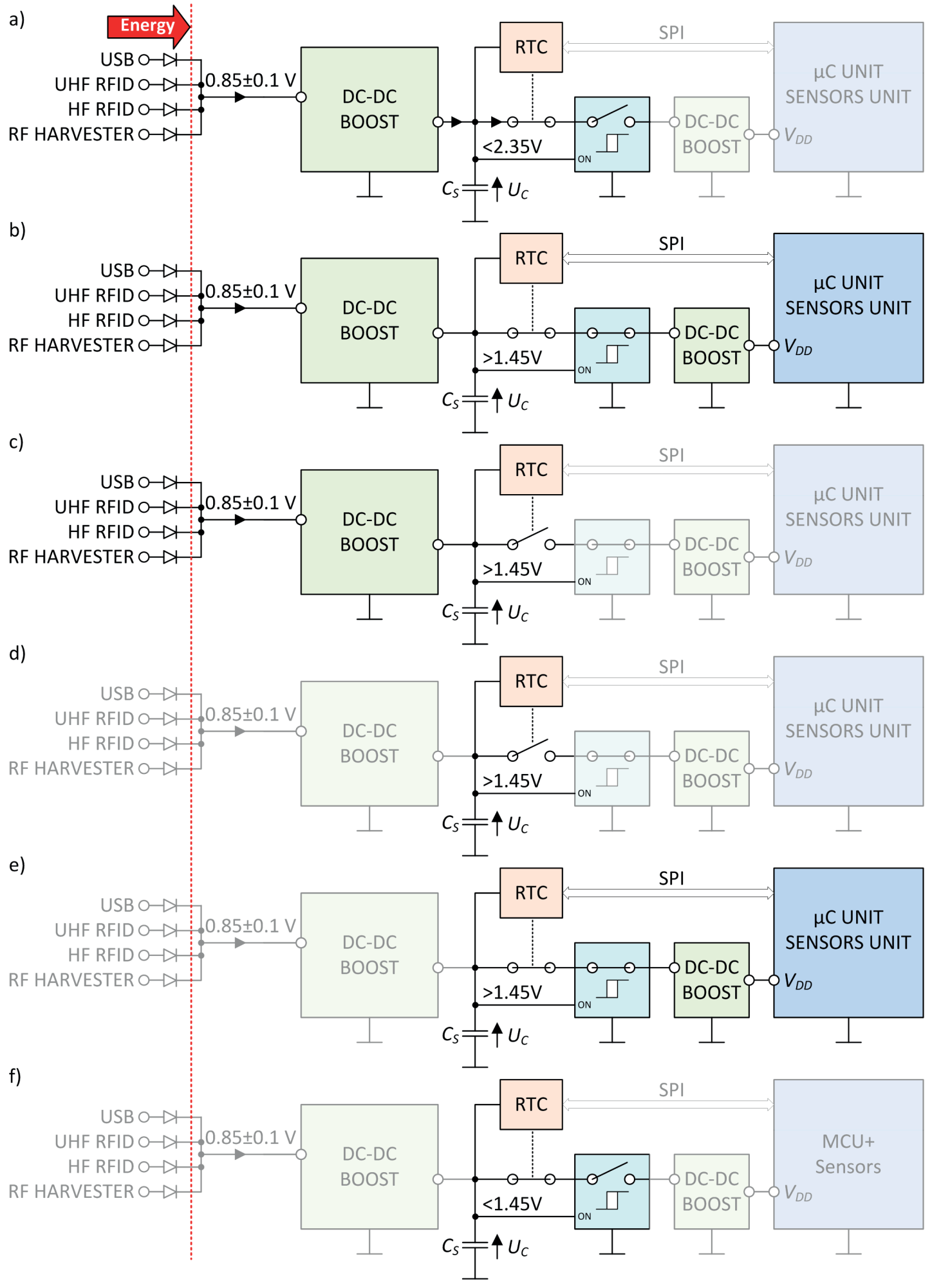

Fig. 5. ESU operation steps: a) charging, b) cold start, c) sleep with charging, d) sleep, e) measurement, f) cut off 
of uselessness energy $E_{\text {off }}$ in the capacitor that can be described by the formula:

$$
E_{\text {off }}=\frac{C_{S} \cdot U_{\text {Coff }}^{2}}{2}=\frac{25 \mathrm{mF} \cdot(1.45 \mathrm{~V})^{2}}{2}=26.28 \mathrm{~mJ}
$$

The very important problem at the design stage is to find adequate integrated circuits and passive elements that are characterised by ultra low quiescent and leakage current. Continuous progress in the silicon technology brings us closer and closer to the zero value of these parameters but in the contemporary commercial electronics this current is so high that the super-capacitor loses gathered energy even if the whole demonstrator is in the sleep mode. So the process of energy harvesting should be permanent.

The stored energy allows demonstrator to work autonomously and it is utilized to acquire information about surroundings of electronically market objects. It is achieved by measuring periodically physical quantities and storing results in the data memories of the RFID chips. The block of signal transducers and conditioning circuits is integrated in the module and consists of 3-axis MEMS accelerometer (analog devices ADXL362), humidity and temperature sensor (Silicon Labs Si7020) and ambient light sensor (Maxim Integrated MAX44009), (Table 1). There is also possibility to connect external intelligent sensors by $\mathrm{I} 2 \mathrm{C}$ bus and it could be useful when the demonstrator is adjusted to future user's applications.

Table 1

Technical parameters of sensors

\begin{tabular}{l|l}
\hline Integrated circuit & Measurement parameters \\
\hline ADXL362 & $\begin{array}{l}\text { Acceleration measured in three ranges: } \\
\pm 2,4,8 \mathrm{~g} \text { with an accuracy of } \pm 10 \%\end{array}$ \\
\hline Si7020 & $\begin{array}{l}\text { Temperature measured in the range of } \\
-40 \ldots+125^{\circ} \mathrm{C} \text { with an accuracy of } \pm 0.4^{\circ} \mathrm{C} \\
\text { Humidity measured in the range of } 0 \ldots 80 \% \\
\text { with an accuracy of } \pm 4 \%\end{array}$ \\
\hline MAX44009 & $\begin{array}{l}\text { Light intensity measured in the range of } \\
0.045 \ldots 180000 \text { lux with an accuracy of } \pm 15 \%\end{array}$ \\
\hline
\end{tabular}

The demonstrator activity is controlled by the control block that is designed on the basis of 32-bit microcontroller (STM32L151RBT6). The demonstration software is elaborated to support a future user in development works with the presented autonomous semi-passive transponder and it can be adapted to the target application requirements by USB interface.

\section{Assembly process of preproduction batch}

The preproduction batch of the demonstrators was manufactured in an automated assembly line of ELMAK Ltd. Company according to the elaborated model of the development board (Fig. 6). The process consisted of three main stages: a) dispensing solder paste, $b$ ) placing components on PCB board and c) reflow soldering.
The first stage was realised by using the manual stencil/ screen printing machine (Uniprint ML) with the mechanically guided squeegee (Fig. 6a). The stencil was designed in the GERBER format and laser-cut in metal sheets of $150 \mu \mathrm{m}$ thickness. The second stage was performed on the Pick \& Place machine for the SMD automated mounting (i-Pulse M20 Yamaha; Fig. 6b). Finally, the assembled PCB boards ware soldered in the reflow oven for medium volume (Esemtec RO400FC-C). The 30 fully functional specimens were obtained as the result of preproduction investigations (Fig. 6c). Since the Pb-free soldering pastes were used, the restriction of European Union directive (Restriction of Hazardous Substances ROHS 2011/65/ UE) was met.

The preproduction batch provided as ready-to-use development set confirms that the elaborated demonstrator can be reproduced on an industrial scale. On the basis of it, the operating parameters of the autonomous semi-passive transponders can be evaluated. It will enable in the future, that dynamic and anticollision identification processes to be effectively conducted.

a)

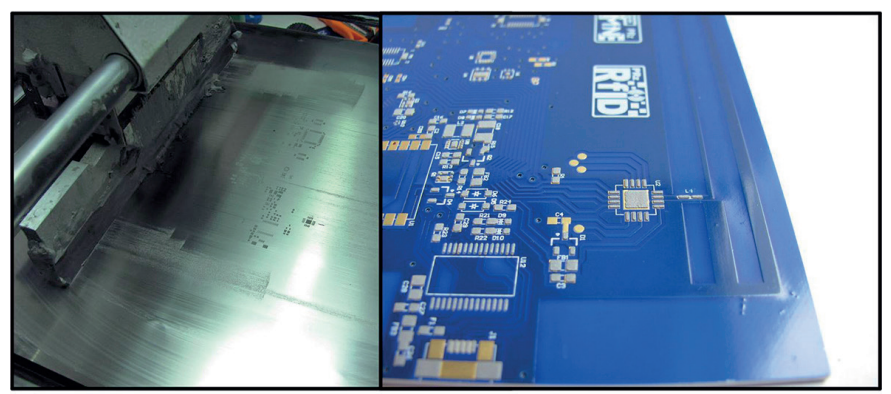

b)
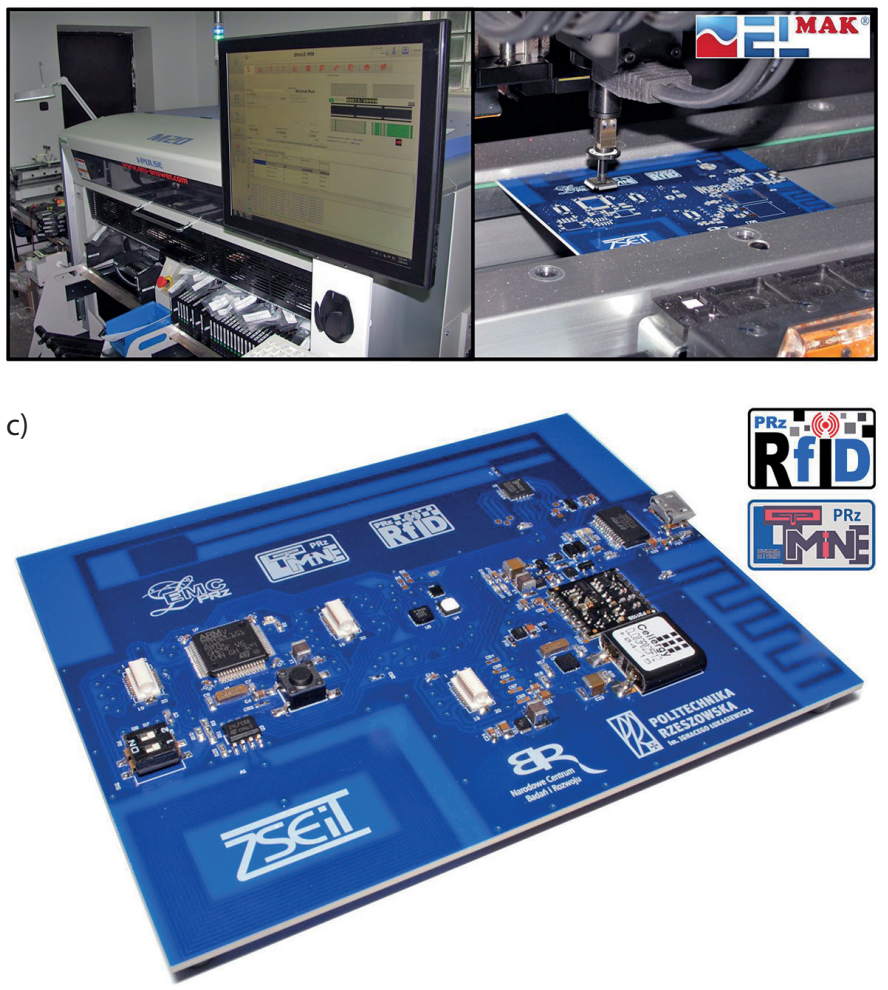

Fig. 6. Assembly process of PCB 


\section{Conclusions}

The conception and practical realisation of the battery-less demonstrator dedicated to develop technique of multiband autonomous semi-passive RFID transponders is presented in the paper. The mechanisms of harvesting energy from different electromagnetic fields, energy conditioning and storing for performing extra untypical functions in automated systems are implemented in the elaborated construction. The worked out development board allows potential users to start investigations and different application projects in the scope of both the object identification as well as the monitoring of environmental conditions. The undertaken enterprises should quickly lead the users to the product commercialization. In this context, the first preproduction batch was assembled in the production line of ELMAK Sp. z o.o. Company. Also, the project concerning the estimation of decision-making determinants in the process of the transponder implementation in the Polish industry was finished and the result are described in detail in [24, 25]. Furthermore, authors of the papers assess the product potential in the market and the possibility of its commercialization.

Acknowledgements. This work was supported in part by the Polish National Centre for Research and Development (NCBR) under Grant No. PBS1/A3/3/2012. The work was developed by using the equipment purchased in the Operational Program Development of Eastern Poland 2007-2013 of the Priority Axis I Modern Economics of Activity I.3 Supporting Innovation under Grant No. POPW.01.03.00-18-012/09-00 and the Program of Development of Podkarpacie Province of The European Regional Development Fund under Grant No. UDA-RPPK.01.03.00-18-003/10-00. The authors acknowledge the ELMAK Company for giving the access to automatic production line where the preproduction batch was assembled.

\section{REFERENCES}

[1] A. Ustundag (Ed.), The Value of RFID - Benefits vs. Costs, Springer-Verlag, 2013.

[2] R. Das and P. Harrop, RFID Forecasts, Players and Opportunities 2014-2024, Report, IDTechEx, 2014.

[3] K. Finkenzeller, RFID Handbook, 3rd Ed., Wiley, 2010.

[4] P. Jankowski-Mihułowicz, G. Pitera and M. Węglarski, "The impedance measurement problem in antennas for RFID technique", Metrol. Meas. Syst. XXI (3), 509-520 (2014).

[5] P. Jankowski-Mihułowicz and M. Węglarski, "Determination of passive and semi-passive chip parameters required for synthesis of interrogation zone in UHF RFID systems", Elektronika ir Elektrotechnika 20 (9), 65-73, 2014.

[6] D. E. Brown, RFID Implementation, McGraw-Hill, 2007.

[7] N. Bartneck, V. Klaas, and H. Schönherr, Optimizing Processes with RFID and Auto ID, Publicis Publ., 2009.

[8] P. Jankowski-Mihułowicz, W. Kalita, M. Skoczylas and M. Węglarski, "Modelling and design of HF RFID passive transponders with additional energy harvester", International Journal of Antennas and Propagation 2013, ID: 242840, 1-10 (2013).
[9] C. W. Lee, S. J. Lee, M. Kim, Y. Kyung and K. Eom, "Capacitive humidity sensor tag smart refrigerator system using the capacitive to voltage converter (CVC)", Int. J. Adv. Sci. Technol. 36, 15-25 (2011).

[10] E. Abad, B. Mazzolai, A. Juarros, D. Gómez, A. Mondini, I. Sayhan, A. Krenkow and Th. Becker, "Fabrication process for a flexible tag microlab", Proc. SPIE 6589, 658900 (2007).

[11] D. Cartasegna, A. Cito, F. Conso, A. Donida, M. Grassi, L. Malvasi, G. Rescio and P. Malcovati, "Smart RFID label for monitoring the preservation conditions of food", Sens. Microsys. 54, 381-385 (2010).

[12] A. Oprea, J. Courbat, N. Bârsan, D. Briand, N. F. de Rooij and U. Weimar, "Temperature, humidity and gas sensors integrated on plastic foil for low power applications", Sens. Actuators B: Chem. 140 (1), 227-232 (2009).

[13] L. Yang, A. Rida, T. Wu, S. Basat and M. M. Tentzeris, "Integration of sensors and inkjet-printed RFID tags on paper-based substrates for UHF cognitive intelligence applications", Proc. IEEE-APS Symp., Honolulu, USA, 1193-1196 (2007).

[14] A. Tani, M. Ugaji and Y. Yamabe, "Building structural-performance monitoring system using RFID tag with sensors", Proc. Int. Conf. Comput. in Civil and Build. Eng., Nottingham, UK, 221 (2010).

[15] P. Jankowski-Mihułowicz, D. Kawalec and M. Węglarski, "Antenna design for semi-passive UHF RFID transponder with energy harvester", Radioengineering 24 (3), 722-728 (2015).

[16] CEPT/ECC, ERC recommendation 70-03 relating to the use of short range devices (SRD), 2014.

[17] H. Yu, J. Wu, L. Fan, Y. Lin, K. Xu, Z. Tang, C. Cheng, S. Tang, J. Lin, M. Huang and Z. Lan, "A novel redox-mediated gel polymer electrolyte for high-performance supercapacitor", Journal of Power Sources 198, 402-407 (2012).

[18] Z.-S. Wua, G. Zhoua, L.-C. Yina, W. Rena, F. Lia and H.-M Cheng, "Graphene/metal oxide composite electrode materials for energy storage", Nano Energy 1 (1), 107-131 (2012).

[19] M. Jayalakshmi and K. Balasubramanian, "Simple capacitors to supercapacitors - an overview", Int. J. Electrochem. Sci. 3, 1196-1217 (2008).

[20] M. Belleville, E. Cantatore, H. Fanet, P. Fiorini, P. Nicole, M. Pelgrom, C. Piguet, R. Hahn, C. van Hoof, R. J. M. Vullers and M. Tartagni, Energy Autonomous Systems: Future Trends in Devices, Technology, and Systems, CATRENE, 2009.

[21] R. J. M. Vullers, R. van Schaijk, I. Doms, C. Van Hoof and R. Mertens, "Micropower energy harvesting", Solid-State Electronics 53 (7), 684-693 (2009).

[22] P. Jankowski-Mihułowicz, W. Lichoń, G. Pitera and M. Węglarski, "Determination of the material relative permittivity in the UHF band by using $\mathrm{T}$ and modified ring resonators", International Journal of Electronics and Telecommunications, 62 (2), 129-134 (2016).

[23] V. Boitier, P. Durand Estèbe, R. Monthéard, M. Bafleur and J. M. Dilhac, "Under voltage lock-out design rules for proper start-up of energy autonomous systems powered by supercapacitors", J. Phys.: Conf. Ser. 476 (1), 012121 (2013).

[24] M. Jankowska-Mihułowicz and K. Chudy-Laskowska, "Cognitive limitations in making investment decisions in Polish enterprises", Proc. 7th Int. Conf. Economic Challenges in Enlarged Europe, A. Hazak (Ed.), Tallinn, Estonia, 1-15 (2015).

[25] M. Jankowska-Mihułowicz, M. Gębarowski, B. Ziółkowski and K. Chudy-Laskowska, "Determinants of investment decision-making in the RFID area in Poland", Proc. 7th Int. Conf. Economic Challenges in Enlarged Europe, A. Hazak (Ed.), Tallinn, Estonia, 1-15 (2015). 